\section{- Chancen für den Fluss}

Nach Ansicht des NABU steht der energiepolitische Nutzen der kleinen Wasserkraft in keinem Verhältnis zu den damit verbundenen ökologischen Schäden. Daher kann und darf ein Ausbau nur über Modernisierungsmaßnahmen an bestehenden Anlagen vollzogen werden, wenn parallel zur Modernisierung eine ökologische Aufwertung stattfindet. Diese Forderung wird vom neuen EEG aufgegriffen, das als erstes Energiegesetz Bezug auf wasserrechtliche Regelungen nimmt. Dies ist eine historische Chance, die Konflikte um die Wasserkraftnutzung zu überwinden, da das neue EEG die Vergütung des aus Wasserkraft gewonnenen Stroms an ökologische Kriterien koppelt. Ein Schritt, der nicht nur aus ökologischer Sicht sinnvoll ist, sondern als rechtliche Maßgabe der WRRL und der nationalen Wassergesetze Beachtung finden muss.

Bezüglich Laufwasserkraftanlagen bis $500 \mathrm{~kW}$ muss bei der Genehmigung von Neuanlagen $a b$ 2008 ein guter ökologischer Zustand oder eine ökologische Verbesserung gegenüber dem Vorzustand des Gewässers nachgewiesen werden. Ebenfalls ab 2008 gilt für diese Anlagen die Auflage, dass neu in Betrieb zu nehmende Anlagen einen räumlichen Zusammenhang zu einer ganz oder teilweise bestehenden Staustufe beziehungsweise Wehranlage aufweisen müssen. Dies bedeutet, dass ab 2008 keine Wasserkraftanlagen bis zu einer Leistung von $500 \mathrm{~kW}$ an unverbauten Standorten mehr genehmigt werden und an Standorten mit einer bestehenden Querverbauung auch nur dann, wenn der gute ökologische Zustand oder eine ökologische Verbesserung gemäß WRRL attestiert ist. Als Nachweis gilt die Vorlage der behördlichen wasserrechtlichen Zulassung. Allerdings wäre eine Funktionskontrolle der Wanderhilfen zur Sicherstellung der Durchgängigkeit wünschenswert, da die Erfahrung zeigt, dass auch schön angelegte Umgehungsgerinne aus hydraulischen Gründen nicht immer funktionieren. Des Weiteren spielt aus Naturschutzsicht die Naturnähe der Wanderhilfen eine große Rolle. Es bleibt, auf eine strenge Auslegung der WRRL und der in Deutschland geltenden wasserrechtlichen Gesetze zu hoffen.

\section{Fazit}

Auch heute müssen zu genehmigende Wasserkraftanlagen schon den ökologischen Standards des europäischen und deutschen Wasserrechts genügen, so dass mit der Aufnahme der ökologischen Kriterien kein Neuland betreten wird, sondern dies nur mit der Vergütung in Zusammenhang gesetzt wird. Das neue EEG schreibt mit einer Vergütung von 9,67 Eurocent für Anlagen bis $500 \mathrm{~kW}$ eine um zwei Eurocent höhere Vergütung gegenüber dem Vorgänger-EEG vor, wenn die vorgegebenen Standards eingehalten werden. Auf diesem Weg werden finanzielle Anreize zur Modernisierung der Wasserkraftanlagen geschaffen. Aus Sicht des Naturschutzes ist das neue EEG ein wichtiger und richtiger Schritt, denn die Konflikt- lösung zwischen Wasserkraftbetreibern und $\mathrm{Na}$ tur- und Umweltschutz kann nur in der naturintegrierten Energienutzung liegen.

Entsprechend der WRRL Artikel $1 \mathrm{a}$ ist eine weitere Verschlechterung der Gewässer zu vermeiden und deren Zustand zu schützen und zu verbessern. Da der Neubau von kleinen Wasserkraftanlagen umweltbelastender sein kann als die Modernisierung bestehender größerer Kraftwerke, greift in diesen Fällen das Verschlechterungsverbot der WRRL. Damit sind Berechnungen des technisch nutzbaren Wasserkraftpotenzials für Neustandorte irrelevant. Nach Ansicht des NABU besteht die Handlungsoption, durch Modernisierung Potenziale an bestehenden Anlagen verstärkt auszuschöpfen, wenn gleichzeitig eine ökologische Verbesserung erzielt wird. In Deutschland gibt es kaum noch weit gehend natürliche Fließgewässer und diese sollten erhalten bleiben.

\section{Die Autorin}

Bettina Keite ist Wasser-Expertin im Fachbereich Naturschutz und Umweltpolitik beim Naturschutzbund (NABU) Bundesverband.

Kontakt: NABU, Herbert-Rabius-Str. 26,

53225 Bonn. Tel. 0228-4036173,

E-Mail: Bettina.Keite@NABU.de

\title{
Die Sonnenseiten der Landschaft
}

\section{Solaranlagen brauchen Platz, sonst können sie keine Energie erzeugen. Mit ökologisch verantwortlichen Solarparks können Freiflächen sogar aufgewertet werden.}

F 1 Von Nikolaus Krane ür die erfolgreiche Realisierung eines umweltgerechten Solarparks ist von entscheidender Bedeutung, Anwohner und regionale Umweltinitiativen möglichst frühzeitig und umfassend über das Projekt zu informieren. Transparente Kommunikation und ein konstruktiver Dialog führen zu deutlich höherer Akzeptanz bei den Bürgern. Um Standortentwicklung und spätere Kapitaleinwerbung für Solarfonds erfolgreich durchführen zu können, ist es für den Durchbruch der Photovoltaik notwendig, dass viele Bürger vor Ort hinter dem Projekt stehen. Zum einen ist eine positive Aufnahme der Solaranlage bei den Anrainern fördernd für die allgemeine Akzep$\tan z$ des Solarparks wie auch künftiger Projekte. Bei den 13 bisher von der voltwerk realisierten Solarprojekten hat sich darüber hinaus gezeigt, dass viele Anwohner aus der näheren Umgebung des Solarparks den Solarprojekten sehr aufge- schlossen gegenüberstehen und sich finanziell daran beteiligen. Aus diesem Grund hat die voltwerk gesonderte Konditionen für die Bürger rund um die Solarparks erarbeitet.

Solare Großprojekte sind für die Weiterentwicklung der umwelt-freundlichen Zukunftstechnologie Photovoltaik zwingend notwendig, um den Einstieg in die Massenproduktion zur Kostensenkung der Komponenten voranzutreiben. Von dieser Entwicklung profitieren gerade auch viele Tausend Betreiber privater Solardächer. Dank der ansteigenden Produktionsmengen der Hersteller können sie künftig mit niedrigen Preisen für Solarmodule rechnen. Doch die Suche nach geeigneten Großdächern gestaltet sich recht schwer. Die Solartechnik stellt hohe Anforderungen an große Dachflächen. Einerseits müssen die Dächer statisch geeignet sein. Zudem müssen sie für die nächsten 20 Jahre zur Verfiigung stehen, eine Bindung, auf die sich Dacheigner oft nicht einlassen. 


\section{- Faktor Freifläche}

Im Zusammenhang mit der Realisierung von Photovoltaikanlagen auf Freiflächen gibt es häufig noch viele offene Fragen und manchmal auch Vorurteile. Zum Teil wird befürchtet, dass die Solaranlagen die vorhandenen Flächen versiegeln würden. Vom Boden selbst werden jedoch etwa nur drei Prozent netto durch die Gestelltechnik belegt. Regen kann nahezu ungehindert auf dem gesamten Gelände versickern. Brutto wird nur etwa ein Drittel der Fläche mit Solarmodulen versehen, da durch die hinter den Modulen auftretende Verschattung großzügige Abstände eingehalten werden. Unter den Modulreihen führt der Lauf der Sonne zu wechselnden Lichtverhältnissen, von denen Flora und Fauna sogar profitieren.

Durch die Abschattung der Module entstehen bereits nach kurzer Zeit verschiedene Kleinklimate. Dies fördert die Struktur und somit die Artenvielfalt. Und bietet zudem noch den Vorteil der Doppelnutzung. Eine naturnahe Fläche, etwa eine Wiese, kann als Weidefläche für Tiere genutzt werden; gleichzeitig wird Strom ohne Schadstoffausstoß produziert. Eine von der RWE in Auftrag gegebenen Studie kommt unter anderem zu dem Ergebnis, dass ,das Potential der Freiflächenanlagen, ehemalige Nutzflächen durch eine solare Stromerzeugung in Partnerschaft mit der Natur aufzuwerten, beim Ausbau der Photovoltaik mit berücksichtigt werden sollte" (1). Dementsprechend weiden zum Beispiel auf den Flächen der von der voltwerk realisierten Solarparks in Hemau und Sonnen jeweils circa fünfzig Schafe und tragen somit zur natürlichen Nutzung der zuvor brach liegenden Flächen bei.

Des Weiteren wird bei Freiflächenanlagen vermutet, dass sie das Landschaftsbild stören könnten. Mit einer maximalen Höhe von vier Metern sind die Modultische nicht von weitem sichtbar. Mit einer Hecke oder einer Baumreihe rund um den Solarpark kann die Einsicht oft sogar völlig verhindert und die Anlage harmonisch in das Landschaftsbild mit eingebunden werden. Zudem gibt es diverse Flächen, die aus landschaftspflegerischer Sicht durch die Errichtung eines Solarparks sogar aufgewertet werden, wie zum Beispiel militärische Liegenschaften, Kohlegruben, kontaminierte Flächen, Klärschlammtrocknungsanlagen oder Mülldeponien.

Für den Solarpark Hemau wurde von der voltwerk die Fläche eines ehemaligen Munitionsdepots der NATO umgewandelt. Das 18 Hektar große ehemalige Militärgelände liegt in einem Waldgebiet innerhalb des „Naturparks Alt- mühltal“, etwa drei Kilometer südwestlich der Stadt Hemau. Gut ein Drittel ist Gehölz und Wald, sämtlich ohne Arten der Roten Liste. Etwa 17 Prozent des Gesamtgebiets waren bereits mit asphaltierten Straßen und Gebäuden belegt. Um von Anfang an auf eine breite Unterstiitzung bauen zu können, wurden bereits vor Baubeginn viele Beteiligte und Bürger mit in die Planung einbezogen. Die Bürger konnten sich im Rahmen verschiedener Informationsabende über Planung, Ausgestaltung und später auch über den Fortschritt des Solarparks informieren. Der Gemeinderat wurde im Rahmen seiner Sitzung- und Gremienarbeit mit einbezogen. Zudem wurde das Projekt in stetigem Austausch mit der Unteren Naturschutzbehörde (UNB) realisiert.

Gerade ein umweltfreundliches Projekt wie der klimaschonende Solarpark Hemau soll die Natur entlasten und nicht beeinträchtigen. Bereits vor Baubeginn wurde ein umfangreicher landschaftspflegerischer Begleitplan für den Bau dieses Solarparks erarbeitet. Ziel war eine qualitative Aufwertung des Geländes für die heimische Flora und Fauna. In enger Abstimmung mit den verantwortlichen Naturschutzbeauftragten, der Oberforstdirektion Regensburg, dem Forstamt Parsberg, der UNB, der Bauabteilung des Landratsamt (LRA) Regensburg und dem Bürgermeister der Stadt Hemau wurde ein umweltverträgliches Konzept nach Artikel 6 des Bayerischen Naturschutzgesetzes zur Umwandlung des ehemaligen Munitionsdepots in einen Solarpark entwickelt.

\section{- Landschaftspflege}

Umfangreiche Maßnahmen zur Landschaftspflege wurden auf dem ehemaligen Munitionsdepot durchgeführt. Obwohl die Gestellsysteme für die gesamte 9,33 Hektar Solarmodulfläche inklusive Zwischenräume nur etwa drei Prozent der Fläche belegen, wurde eine Ausgleichsfläche von 6,4 Hektar zur ökologisch wertvollen Nutzung freigegeben und rekultiviert. Der Anteil der wertvollen, so genannten 13d-Flächen, wurde von 17,2 auf 40,2 Prozent erhöht, die seggenreiche Feuchtfläche erhöhte sich von 9,8 auf 15 Prozent. Das Flachmoor wurde nicht bebaut. Ein Teil der vorhandenen Asphaltstraßen wurde mit sandigem Oberboden überdeckt und konnte sich so zu mageren und frischen Glatthaferwiesen entwickeln. Die Tierwelt profitiert mittlerweile von den zusätzlichen Flachwasserzonen für Amphibien und Lurche. Die Vogelwelt freut sich über die neuen Waldränder und Wiesen. Eine fachliche Begleitung der Neuanlage und Pflege der Fläche über fünf Jahre sichert die erfolgreiche Umwandlung des Geländes. Der Solarpark Hemau wird für zwanzig Jahre von der voltwerk AG betrieben. Das Erneuerbare Energien Gesetz (EEG) garantiert den Betreibern von Stromanlagen für diesen Zeitraum die Abnahme des umweltfreundlich produzierten Stroms zu festen Vergütungssätzen.

Dies ist ein Plus an Planungssicherheit, das die über Fonds am Solarpark beteiligten Investoren überzeugt. Zugleich bedeutet dies, dass das Engagement der voltwerk AG nach dieser Zeit enden wird. Die derzeit eingesetzten Module können jedoch aufgrund ihrer Qualität weit über dreißig Jahre umweltfreundlichen Strom produzieren. Um dieses Potential auszunutzen und den Fortbestand des Solarparks zu gewährleisten, wurde gleich zu Projektbeginn vertraglich festgelegt, dass der Solarpark durch die Servicegesellschaft für regenerative Energieprojekte weiter betrieben wird.

Freiflächenanlagen sind und bleiben bei der Realisierung von solaren Großprojekten eine wichtige Möglichkeit. Voraussetzung ist dabei, dass sich die Flächen dafür eignen und sich der Solarpark landschaftlich integrieren lässt. So realisiert die voltwerk AG derzeit im saarländischen Göttelborn ein neues Großprojekt. Der 4-Megawatt-Solarpark entsteht auf dem Gelände einer ehemaligen Kohlegrube, einem Brachgebiet, das aufgrund seiner industriellen Vergangenheit bislang kaum andere Nutzungsmöglichkeiten zuließ. Freiflächenprojekte bieten die Möglichkeit eine Wertschöpfung mittels umweltfreundlicher Stromgewinnung zu erreichen und gleichzeitig ehemalige Nutzflächen aufzuwerten. Diesen Doppelnutzen gilt es zu erkennen und weiterhin verantwortungsvoll nutzen.

\section{Anmerkung}

(1) Teggers-Junge, S.: Schattendasein und Flächenversiegelung durch Photovoltaik. Essen 2004.

\section{Der Autor}

Nikolaus Krane ist Vorstandsvorsitzender der voltwerk AG.

Kontakt: voltwerk AG, Anckelmannsplatz 1 20537 Hamburg. E-Mail: info@voltwerk.de 
(c) 20I0 Authors; licensee IÖW and oekom verlag. This is an article distributed under the terms of the Creative Commons Attribution Non-Commercial No Derivates License (http://creativecommons.org/licenses/by-nc-nd/3.o/), which permits unrestricted use, distribution, and reproduction in any medium, provided the original work is properly cited. 